Colin R. Chilvers B MED DA (US) FANZCA,* Alison Goodwin MB FCA,

H imat Vaghadia M B BS MHSC FRCPC FFARCS, ${ }^{*} \dagger$

G.W.E. M itchell MB CHB FRCOG FRCS(ed) FRCSC $\neq$

\title{
Selective spinal anesthesia for outpatient laparoscopy. V: Pharmacoeconomic comparison vs general anesthesia
}

Purpose: To compare the cost and effectiveness of small-dose spinal anesthesia (SP) with general anesthesia (GA) for outpatient laparoscopy.

M ethods: A retrospective record analysis of 24 patients who received SP were compared with 28 patients who received GA in our Daycare centre. The costs of anesthesia and recovery were calculated, from an institutional perspective, using 1997 Canadian Dollar values. Effectiveness was measured in terms of time for anesthesia and recovery, and postoperative antiemetic and analgesic requirements.

Results: Both groups were well matched for age, weight, duration and type of surgery. The mean total cost for the SP group of $\$ 53.45 \pm 10.40$ was no different from that for the GA group of $\$ 48.92 \pm 10.25(95 \% \mathrm{Cl}$ $-10.3,1.2)$. Time to administer anesthesia was longer in the SP group with a mean time of $18 \pm 8$ min compared with $10 \pm 3$ min in the GA group $(\mathrm{Cl}-11.3,-4.7)$. Recovery time in the PACU was longer in the SP group 123 \pm 51 min compared with $94 \pm 48$ min $(\mathrm{Cl}-56.6,-1.4)$. Postoperative antiemetic requirements were similar: $8 \%$ in SP group vs $14 \%$ in GA group, whereas analgesic requirements were less in the SP group with $25 \%$ receiving analgesia compared with $75 \%$ in the GA group $(P<0.05)$.

Conclusion: The total cost of anesthesia and recovery using SP is similar to that for GA when used for outpatient laparoscopy. Spinal anesthesia was less effective than GA in time to administer anesthesia and in duration of recovery. Postoperative analgesic requirements were reduced using SP.

O bjectif : Comparer le coût et l'efficacité de la rachianesthésie à faibles doses (RA) avec ceux de l'anesthésie générale (AG) pour la laparoscopie ambulatoire.

M éthode : U ne analyse rétrospective des dossiers de 24 patients qui ont reçu une RA a été comparée à celle des dossiers de 28 patients qui ont reçu une AG à notre centre ambulatoire. Le coût de l'anesthésie et de la récupération a été calculé, d'un point de vue institutionnel, en utilisant les valeurs en dollars canadiens. L'efficacité a été mesurée selon le temps dévolu à l'anesthésie et à la récupération et selon les demandes postopératoires d'antiémétiques et d'analgésiques.

Résultats : L'appariement des deux groupes a été réalisé suivant l'âge, le poids, la durée et le type d'intervention chirurgicale. Le coût total moyen dans le groupe RA, de 53,45\$ $\pm 10,40$, était semblable dans le groupe AG, de $48,92 \$ \pm 10,25$ (IC $95 \%-10,3 ; 1,2$ ). Le temps précédant l'administration de l'anesthésie a été plus long dans le groupe RA, le temps moyen a été de $18 \pm 8$ min comparé à $10 \pm 3$ min dans le groupe AG (IC $-11,3$; $-4,7)$. La récupération en salle de réveil a été plus longue dans le groupe RA, $123 \pm 51$ min comparée à $94 \pm$ 48 min (IC $-56,6 ;-1,4)$. La demande d'antiémétiques a été similaire : $8 \%$ pour la RA vs $14 \%$ pour l'AG, tandis que la demande d'analgésiques a été moindre dans le groupe RA, $25 \%$ comparé à $75 \%$ dans le groupe AG $(P<0,05)$.

Conclusion : Le coût total de l'anesthésie et de la récupération est similaire, qu'on utilise la RA ou l'AG, dans le cadre de la laparoscopie ambulatoire. La rachianesthésie a été moins efficace que l'AG si on considère la période préanesthésie et la récupération plus longues dans ce groupe. Les besoins d'analgésiques postopératoires sont réduits avec la RA.

From the D epartments of Anaesthesia,* H ealth Care and E pidemiology, $\dagger$ and Gynaecology, $¥$ Vancouver General H ospital, U niversity of British Columbia, Vancouver, British Columbia, Canada.

Address correspondence to: Dr. H imat Vaghadia, D epartment of Anaesthesia, Room 3200, Vancouver General H ospital, 3rd Floor - 910

West 10th Ave, Vancouver, BC, V5Z 4E 3 Canada. Phone: 604-875-4575; Fax: 604-875-5209; E-mail: vaghadia@interchange.ubc.ca A ccepted for publication October 29, 2000. 
A new technique of spinal anesthesia (SP) using small-dose hypobaric lidocaine-fentanyl solution has been introduced in our institution. Previous studies ${ }^{1,2}$ have validated its effectiveness and acceptability for short duration outpatient laparoscopy, and its faster recovery profile in comparison with conventional dose hyperbaric lidocaine. It was developed as a potentially cheaper and more efficient alternative to general anesthesia (GA) for this common procedure.

The purpose of this study was to compare the direct hospital costs of anesthesia for outpatient laparoscopy using SP with those using GA. Effectiveness was evaluated by comparing time taken for anesthesia induction and recovery, the need for postoperative antiemetics and analgesia, and the incidence of other complications. The hypothesis tested was that SP is cheaper, more efficient, and has fewer complications than GA for outpatient laparoscopy.

\section{M ethods}

At our institution a proportion of patients having gynecological laparoscopy as outpatients have received spinal anesthesia, while the remainder were given more traditional general anesthesia. Patient preference largely determined the choice of technique. A retrospective analysis of patient records was undertaken after approval was obtained from the Clinical Screening Committee for Research I nvolving $\mathrm{H}$ uman Subjects. Records of patients from 1995 were screened by type of anesthesia, type of surgery and name of surgeon. U Itimately, a comparison was performed between 28 patients given SP and 28 who received GA for laparoscopic sterilisation. All cases were matched by year of surgery, same surgeon and same operation to ensure minimal impact from changes in anesthetic or surgical techniques.

The following demographic data were obtained from the patients charts: age, weight, duration of surgery, total time spent in $O R$, and surgical procedure. The record of supplies, drugs, and gases used during anesthesia and recovery was collated to calculate costs. The time taken to administer anesthesia, time spent in the Post Anesthetic Care U nit (PACU), and the time taken from arrival in PACU until discharge home were analyzed to compare the effectiveness of the two techniques. Sub components of these times are not recorded in the charts and could not be determined. Since the incidence of nausea and vomiting and the degree of pain experienced postoperatively by patients cannot be determined from records, the administration of antiemetics and analgesics while in hospital was used to compare the magnitude of these complications.
Costs of anesthesia and recovery for each group were calculated in Canadian Dollars at 1997 values. All values refer to the direct expense to the hospital and not what the patient is charged. The study perspective was strictly institutional. Costs were assumed to be constant during the period of study.

"Supplies" included all disposables used in the administration of anesthesia and recovery care and included items such as cannulas, oxygen masks, intravenous tubing and fluids. It was assumed that one syringe and needle of the appropriate size were used for each drug administered. Sterilizing of airway and breathing equipment involved labour and packaging and these costs were calculated by the Sterile Supply D epartment.

D rug costing was performed with the assumption that residual drug left in single use ampoules was dis carded. "Gases" includes the cost of isoflurane calculated using the formula published by $D$ ion. ${ }^{3} O$ xygen and nitrous oxide costs were calculated using the cost per litre of bulk supply to the hospital. The amount of oxygen used as the driving gas for ventilators was derived from multiplying the duration of surgery and the manufacturer's estimate of consumption. Fresh gas flows were not recorded on over half of the charts analyzed. In order to calculate costs, the mean flow used in a contemporary study in our D aycare centre ${ }^{4}$ was substituted for missing values. This approach closely reflects actual clinical use of fresh gases in our centre.

The average cost of providing one hour of a registered nurse's time in our Daycare centre in 1997 was $\$ 31.12$. This figure takes into account leave and benefit entitlements. The average nurse to patient ratio and the time a patient spent in the recovery room were used to calculate the nursing cost of recovery.

The cost of nursing time in the operating theatre was not calculated, as the time spent in theatre was the same for both groups. Likewise, other costs that were common to both groups such as capital depreciation, physician reimbursement, maintenance of the centre, linen and catering for patients, etc., were not analysed.

During the study period, anesthetic, surgical and nursing staff members were the same. N ursing protocols and discharge criteria from the PACU (orientated; stable vital signs > 30 min; no surgical complications; absence of side effects; pain controlled with oral analgesia; absence of motor block; sensory block S3; able to ambulate) were the same for both groups. Staffing in the PACU was also unchanged in terms of nurse to patient ratio. The PACU functions as an integrated unit so that both Phase I and Phase II patients are nursed in the same area.

Statistical analysis was performed by the hospital epidemiology and statistics department using the 
SPSS program. Data are summarized as means, SD and $95 \%$ confidence intervals. $P<0.05$ was considered significant.

Results

All patients in the SP group received a subarachnoid block with a hypobaric solution of lidocaine $(2-2.5 \mathrm{ml}$ of $1 \%)$ and fentanyl (10-25 $\mu \mathrm{g})$ administered via a 27 gauge Whitacre needle. All blocks provided good surgical anesthesia of the abdomen. Glycopyrrolate was administered to $92 \%$ to prevent vasovagal episodes that sometimes occur with the combination of laparoscopy and spinal anesthesia. Intravenous alfentanil was administered to $83 \%$ as prophylaxis for treatment of intraoperative shoulder-tip pain due to its rapid onset, with $8 \%$ requiring both alfentanil and small increments of propofol to treat this.

In the GA group anesthesia was induced with propofol, an opioid (fentanyl in $86 \%$, sufentanil in $11 \%$, not recorded in $3 \%$ ), and a muscle relaxant (mivacurium in $57 \%$, succinylcholine in $32 \%$, none in $3 \%$, not recorded in $6 \%$ ). Endotracheal tubes were inserted in all patients. Anesthesia was maintained with oxygen, nitrous oxide and isoflurane administered by intermittent positive pressure ventilation.

The two groups were well matched in demographic characteristics (T able I). The ASA class was usually not recorded but the medical histories suggested patients in both groups were ASA I-II.

The total cost of anesthesia for both groups was similar (Table II). The higher cost of sterilising, drugs, and gases in the GA group being balanced by the higher cost of supplies in the SP group. T wo items the Whitacre needle and the disposable preparation tray for spinal anesthesia - accounted for most of the supply cost in the SP group. The total costs during recovery for both groups were also similar (Table II). The cost of supplies/ drugs during recovery in the GA group was higher statistically, but the values were so small as not to be of practical importance. O verall, the combined cost of anesthesia and recovery in the SP group was not different from that for the GA group.

E ffectiveness data are shown in Table III. The time taken to administer anesthesia was longer in the SP group. The SP group spent longer in the PACU than the $\mathrm{GA}$ group. There was not difference in time to discharge, although the trend was for the SP patients to take longer.

There was no difference in antiemetic usage between groups but postoperative analgesia was required by fewer patients in the SP than the $G A$ group. O ne patient in the GA group was given $0.1 \mathrm{mg}$ naloxone at the conclusion of surgery. There were no
TABLE I Demographic data

\begin{tabular}{llll}
\hline & $\begin{array}{l}\text { Spinal } \\
(\mathrm{n}=24)\end{array}$ & $\begin{array}{l}\text { General A nesthesia } \\
(\mathrm{n}=28)\end{array}$ & $95 \% \mathrm{Cl}$ \\
\hline Age $(\mathrm{yr})$ & $35 \pm 5$ & $34 \pm 6$ & $-4.1,2.1$ \\
Weight $(\mathrm{kg})$ & $67 \pm 18$ & $64 \pm 14$ & $-11.9,5.9$ \\
Surgical time (min) & $16 \pm 7$ & $20 \pm 11$ & $-1.2,9.2$ \\
Time in OR (min) & $38 \pm 10$ & $34 \pm 8$ & $-3.1,4.2$ \\
Type of surgery & & & \\
L / LS+T & $19 / 5$ & $19 / 9$ & \\
\hline
\end{tabular}

$\mathrm{N}$ on-count values are mean $\pm \mathrm{SD}, * \mathrm{LS}=$ laparoscopic steriliza tion, $\mathrm{T}=$ termination of pregnancy

TABLE II Costs

\begin{tabular}{|c|c|c|c|}
\hline & Spinal & $\begin{array}{l}\text { General } \\
\text { A nesthesa }\end{array}$ & $95 \% \mathrm{Cl}$ \\
\hline \multicolumn{4}{|l|}{ A nesthesia } \\
\hline Supplies & $23.3 \pm 0.08$ & $12.4 \pm 0.09$ & $-10.9,-10.8^{*}$ \\
\hline Sterilizing & 0 & $1.8 \pm 0$ & $*$ \\
\hline Drugs & $10.9 \pm 4.7$ & $16.4 \pm 6.4$ & $2.3,8.8^{*}$ \\
\hline Gases & 0 & $1.6 \pm 1.0$ & $*$ \\
\hline Subtotal & $34.3 \pm 4.8$ & $32.3 \pm 6.6$ & $-5.2,1.3$ \\
\hline \multicolumn{4}{|l|}{ R ecovery } \\
\hline Supplies/ drugs & $0.02 \pm 0.07$ & $0.77 \pm 1.3$ & $0.2,1.3^{*}$ \\
\hline N ursing time & $19.1 \pm 8.3$ & $15.7 \pm 7.6$ & $-7.8,1.1$ \\
\hline Subtotal & $19.1 \pm 8.2$ & $16.5 \pm 7.8$ & $-7.1,1.2$ \\
\hline Grand total & $53.4 \pm 10.4$ & $48.9 \pm 10.2$ & $-10.3,1.2$ \\
\hline
\end{tabular}

Values are mean \pm SD in CAN ADIAN $\$, *=$ significant at $\mathrm{P}<$ 0.05

TABLE III Effectiveness

\begin{tabular}{llll}
\hline & Spinal & $\begin{array}{l}\text { General } \\
\text { Anesthesa }\end{array}$ & $95 \% \mathrm{Cl}$ \\
\hline Anesthesia time (min) & $18 \pm 8$ & $10 \pm 3$ & $-11.3,-4.7^{*}$ \\
PACU time (min) & $123 \pm 51$ & $94 \pm 48$ & $-56.6,-1.4^{*}$ \\
Time to discharge (min) & $150 \pm 59$ & $124 \pm 49$ & $-56.1,4.1$ \\
PACU analgesics: & & & \\
Parenteral opioids & $8 \%$ & $21 \%$ & $*$ \\
O ther & $17 \%$ & $54 \%$ & $*$ \\
None & $75 \%$ & $25 \%$ & $*$ \\
PACU antiemetics & $8 \%$ & $14 \%$ & \\
\hline
\end{tabular}

$\mathrm{N}$ on percentage values are mean $\pm \mathrm{SD}, *=$ significant at $\mathrm{P}<0.05$ PACU $=$ post anesthetic care unit

other complications documented in either group and no patient required overnight admission.

\section{Discussion}

Cost is now an important item to justify use of a particular technique, especially a new one. This study found no difference in total cost between SP and GA for outpatient laparoscopy. This is in contrast to other 
comparisons of regional and GA, where the cost of SP for arthroscopy ${ }^{5}$ and intravenous regional anesthesia (IVRA) for hand surgery ${ }^{4}$ were less than the cost of $G A$. Reasons for this unexpected result were the relatively high cost of supplies needed to perform SP and the failure of SP to reduce the time spent recovering in the PACU.

Studies comparing SP to GA have found shorter, ${ }^{6}$ longer, ${ }^{7}$ and similar ${ }^{5}$ recovery times. This is not surprising considering the different surgery and criteria for block resolution employed. In the current trial, the longer time spent in PACU by SP patients was apparently due to the need to fulfill the discharge criteria for resolution of sensory block rather than from side effects. Despite minimal motor block and a sensory component that wears off faster than conventional dose hyperbaric lidocaine, SP with the small-dose hypobaric lignocaine-fentanyl solution ${ }^{1,2}$ still took longer to resolve than the effects of GA. This is may be improved by other techniques of SP that utilize lower dose of local anesthetic drugs, or other combinations of local anesthetics / opioids. The recovery time could also possibly be lessened if patients were discharged from the PACU with residual sensory block - a notion that has not been tested in the ambulatory care setting. H owever, in the obstetric setting, it is now accepted practice to allow patients the opportunity to ambulate in the presence of a selective neuraxial block which provides sensory analgesia without motor weakness and with intact dorsal column function. In companion studies, we present our preliminary results with the technique of 'walk-in walk-out' selective spinal anesthesia. ${ }^{8,9} \mathrm{H}$ owever, further studies are required before the safety of such practice can be recommended on a wider scale.

Spinal anesthesia was less efficient in time taken to administer anesthesia - this was not unexpected. ${ }^{5}$ $\mathrm{N}$ evertheless, the average difference of eight minutes between time taken to administer SP and GA is not prohibitive, even in the time-constrained day surgery setting.

$\mathrm{N}$ o difference was found in postoperative antiemetic usage, although it was expected to be less in the SP group. ${ }^{6,10} \mathrm{~A}$ larger prospective study would be needed to show if the incidence of nausea and vomiting really is reduced in these patients by using SP. Postoperative analgesic requirements were found to be less in the SP group in line with other comparative studies. ${ }^{5,6}$ Whether this is due to residual sensory block or the analgesic properties of intrathecal fentanyl is unclear. A prospective study to see how long this benefit continues after discharge would be interesting.

The retrospective method used in this study is open to criticism because it is more prone to bias than a prospective randomised trial. Patient preference dictated the choice of anesthetic; this could have influenced the distribution of risks (for example, more patients at risk of emesis opting for spinal anesthesia). $O$ ne advantage of a retrospective design is its ability to generate or refute a hypothesis without incurring the large expenses of a prospective study. The patients in both groups underwent surgery in the same time period, with the same staff and protocols, and had the same demographic characteristics. The essentially negative findings of the study confirm the methodology was sound and unbiased. Since calculation of costs was as detailed as possible, it is unlikely that the occasional missing value or estimation utilised would effected the overall results. The dollar costs cannot be applied to other institutions, although the conclusions should be applicable. In the end the study design and results were largely able to refute the hypothesis, although a prospective trial would provide further useful information on complications after discharge as well as comparative rates of patient satisfaction. An very large study would be required to determine the pharmacoeconomic impact of rare but serious complications of the two techniques (e.g., post dural puncture headache, succinylcholine apnea, etc.).

In conclusion, this study found that the cost for SP for outpatient laparoscopy were similar to those for GA, and that SP took longer to administer and for patients to recover. Spinal anesthesia was effective in reducing postoperative analgesia requirements for this procedure. Although SP is an alternative to GA for individual patients, its widespread use will not reduce costs or improve efficiency of anesthesia and recovery for outpatient laparoscopy. Future studies may have to examine the feasibility and safety of 'fast tracking' patients who receive SP in order to improve its cost effectiveness.

\section{References}

1 Vaghadia H, M LLeod DH, Mitchel GWE, Merrick PM, ChilversCR. Small-dose hypobaric lidocaine-fentanyl spinal anesthesia for short duration oupatient laparoscopy. I. A randomized comparison with conventional dose hyperbaric lidocaine. Anesth Analg 1997; 84: 59-64.

2 Chilvers CR, Vaghadia H, Mitchell GWE, Merrick PM. Small-dose hypobaric lidocaine-fentanyl spinal anesthesia for short duration oupatient laparoscopy. II. O ptimal fentanyl dose. Anesth Analg 1997; 84: 65-70.

3 Dion P. The cost of anaesthetic vapours (Letter). Can J Anaesth 1992; 39: 633.

4 Chilvers $C R, K$ inahan A, Vaghadia $H$, Merrick PM. Pharmacoeconomics of intravenous regional anaesthesia vs general anaesthesia for outpatient hand surgery. Can J Anaesth 1997; 44: 1152-6. 
5 D ahl V, Gierlff C, O mland E, Ræler JC. Spinal, epidural or propofol anaesthesia for out-patient knee arthroscopy? Acta Anaesthesiol Scand 1997; 41: 1341-5.

6 Standl T, Eckert S, Shulte am Esch J. Postoperative complaints after spinal and thiopentone-isoflurane anaesthesia in patients undergoing orthopaedic surgery. Acta Anaesthesiol Scand 1996; 40: 222-6.

7 Tessier MJ, K ardash K, K leiman S, R ossignol M. A retrospective comparison of spinal and general anesthesia for vaginal hysterectomy: a time analysis. Anesth Analg 1995; 81: 694-6.

8 Vaghadia H, Viskari D, Mitchell GWE, Berrill A. Selective spinal anesthesia for outpatient laparoscopy. I: Characteristics of three hypobaric solutions. Can J Anesth 2001; 48: 256-60.

9 Vaghadia H, Solylo M, H enderson C, M itchell GWE. Selective spinal anesthesia for outpatient laparoscopy. II: Epinephrine and spinal cord function. Can J Anesth 2000.; 48: 261-66.

10 Dahl JB, Schultz P, A nker-M ller E, Christensen EF, Staunstrup H G, C arlsson P. Spinal anaesthesia in young patients using a 29-gauge needle: technical considerations and an evaluation of postoperative complaints compared with general anaesthesia. Br J Anaesth 1990; 64: 178-82. 